\title{
Advanced Space Suit Portable Life Support Subsystem Packaging Design
}

\author{
Robert Howe, Chuong Diep, Bob Barnett \\ Hamilton Sunstrand \\ Michael Rouen, Gretchen Thomas \\ NASA Johnson Space Center \\ Jack Kobus \\ A.T. Kearney
}

\begin{abstract}
This paper discusses the Portable Life Support Subsystem (PLSS) packaging design work done by the NASA and Hamilton Sundstrand in support of the 3 future space missions; Lunar, Mars and zero-g. The goal is to seek ways to reduce the weight of PLSS packaging, and at the same time, develop a packaging scheme that would make PLSS technology changes less costly than the current packaging methods. This study builds on the results of NASA's in-house 1998 study, which resulted in the "Flex PLSS" concept. For this study the present EMU schematic (low earth orbit) was used so that the work team could concentrate on the packaging. The Flex PLSS packaging is required to: protect, connect, and hold the PLSS and its components together internally and externally while providing access to PLSS components internally for maintenance and for technology change without extensive redesign impact. The goal of this study was two fold:

1. Bring the advanced space suit integrated Flex PLSS concept from its current state of development to a preliminary design level and build a proof of concept mockup of the proposed design, and; 2. "Design" a Design Process, which accommodates both the initial Flex PLSS design and the package modifications, required to accommodate new technology.

\section{INTRODUCTION}

PORTABLE LIFE SUPPORT SUBSYSTEM

For this discussion, the Portable Life Support System is the space suit life support system when the suit system is designed to be completely independent of other vehicles or support systems. It provides the life support functions of oxygen supply, breathing gas circulation and
\end{abstract}

revitalization, and crew person thermal control. Also embedded in the system is the necessary power supplies to run the PLSS and the PLSS usually hosts the communication radios. Because the PLSS can fail there is usually a secondary system and for our purposes here, the packaging must accommodate that system as well.

\section{ORIGIN OF THE PROBLEM}

The driving issues for this effort are PLSS carry mass and launch mass. Both are important. But the first, carry mass, is probably the more limiting issue. As NASA returns to exploring, an attempt is being made to look as far forward as possible and design systems to meet the far reaching goals as much as possible. From that perspective, Mars carry mass becomes an issue because, even if the transient de-conditioning on the zero-g ride out is ignored or overcome by counter measures, a crewperson working on Mars for any period of time will condition to the Martian gravity. So, the crew people will not be able to carry on Mars any more mass than they can carry on Earth despite the fact that the Mars gravity is lower than Earth gravity ${ }^{1}$. The well established rule of thumb (in backpacker's experience)

\footnotetext{
${ }^{1}$ This statement is not without controversy within NASA. There are some who would expect PLSS carry mass to act as a counter measure and help keep the crewperson conditioned to earth capability. The PLSS design community is working under this assumption and striving to reduce PLSS mass as much as possible because the assumption is conservative, fits with known human physiology, and gives the most system level flexibility. If we don't work the weight of the PLSS down now and if we find later there is a problem we have lost the unrecoverable resource of time to work the problem. This paper will illustrate it is a difficult problem that will require time to overcome. If carry mass proves not to be a problem, there will be plenty of ways we can add carry mass such as added expendables for longer planned or emergency operating time, tools, instruments, cameras etc. all of which will enhance the exploration effort
} 
is that a person can carry about $25-30 \%$ of their lean body mass for a full work day. Since our current suit system equals the person's mass, we face the need to reduce suit system mass by $2 / 3$ or limit the work day. This must be accomplished while attempting to maintain the ability of the package to accommodate changes at minimum cost.

In any mass reduction effort the first question is "What are the heavy items?" In the space suit, the PLSS is the heavy item. And in a Pareto diagram of PLSS item mass, (Figure 1) packaging is the leading contributor to system mass. This fact led NASA to address packaging as a technology that needed development for the specific purpose of reducing mass.

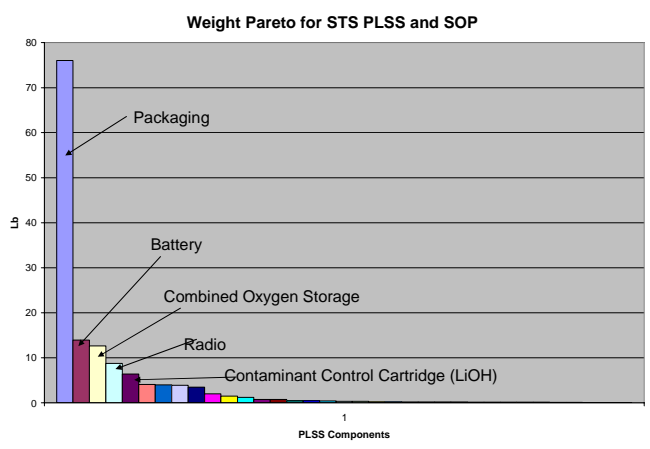

Figure 1 - Shuttle PLSS Weight Pareto Diagram

\section{PACKAGING HISTORY}

In the history of United States human space flight there have been two PLSS packages designed and flown, the Apollo PLSS, the Shuttle PLSS.

The Apollo PLSS was a weight constrained design. In the Apollo PLSS package, the water tank formed the upright leg and the $\mathrm{LiOH}$ cartridge formed the horizontal leg of a "T" structure that was the structural backbone of the package. As the Apollo program progressed, there was a major change to go from a four (4) hour capable PLSS to a seven (7) hour capable PLSS. The LiOH cartridge could accommodate the longer duration by using a slightly larger amount of a more efficient $\mathrm{LiOH}$, but the water tank could not hold the additional water needed. Increasing the water tank size required the addition of an auxiliary reservoir to the side of the PLSS.

The Shuttle PLSS was a volume constrained design. It was designed to meet a fixed front to back dimension to accommodate upper deck access. This design was done with the thought that changes in duration requirements as experienced in Apollo should be easy to accommodate. Consequently, the package was laid out so that the time dependent items (i.e., consumables) were separate from the time independent items (fan, valves, etc.). The package also had the requirement that it be maintainable on the ground between flights. This led to the valve module with its cartridge mounted components. The need for flexibility has also been experienced in the Shuttle program. To accommodate use on the ISS, the PLSS $\mathrm{CO}_{2}$ absorption system was changed to be a regenerable system (METOX) and the battery was upgraded for additional wet shelf life. Both of these components were on the outside of the package; and therefore, could grow in one dimension to allow those changes at the expense of changing the front to back allowable dimension which was allowed since the requirement for upper deck access was no longer in force. In addition, in-flight maintainability has been added by the addition of captive fasteners to the ground maintainable components. But again, in the Shuttle package, the water tank forms the backbone of the structure and a major change in thermal control system would require the package design be significantly redesigned or abandoned for a new design.

The flexibility to allow for technology/mission adaptability is critical for the PLSS due to the varied missions that NASA may need to support in the next 30 years (Figure 2).

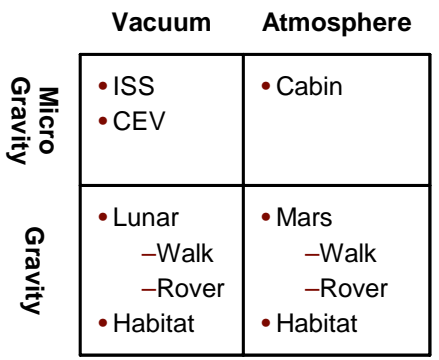

Figure 2 - Mission Types

\section{PRIOR STUDIES}

\section{Packaging Factor Studies}

Two key studies $(1,2)$ quantifying the impact of packaging on system weight have shown that PLSS packaging weighs from 50\% (Orlan-DMA backpack and unit) to $130 \%$ (Shuttle PLSS and Secondary Oxygen Pack (SOP)) as much as the components packaged. The Apollo PLSS packaging weighed $70 \%$ of the components packaged. Here the PLSS is taken to be both the primary and emergency portable life support systems. These studies define mass $\left(\mathrm{PF}_{\mathrm{m}}\right)$ and volume $\left(P F_{v}\right)$ packaging factors which are one way to measure the impact of packaging on the system. $\mathrm{PF}_{\mathrm{m}}$ is the ratio of the total mass of the system to the mass of the nonpackaging components, and $\mathrm{PF}_{\mathrm{v}}$ is the ratio of the total volume of the system to the volume of the nonpackaging components. The first study defined packaging by the following ground rules: 
- Any item performing a major, useful life support function is a component to be packaged and is not packaging.

- Harnesses, connectors, switches, brackets, wiring, and plumbing are packaging.

- Structure is packaging, even in such special cases as the Shuttle valve module housing.

For example, a PLSS that had a total mass of 220 pounds and a non-packaging mass (i.e. component mass) of 100 pounds (oxygen tanks, batteries, etc.) would have a $\mathrm{PF}_{\mathrm{m}}$ of $2.2(220 / 100)$. PLSS designs can be mapped on a volume and mass packaging basis (Figure 3).

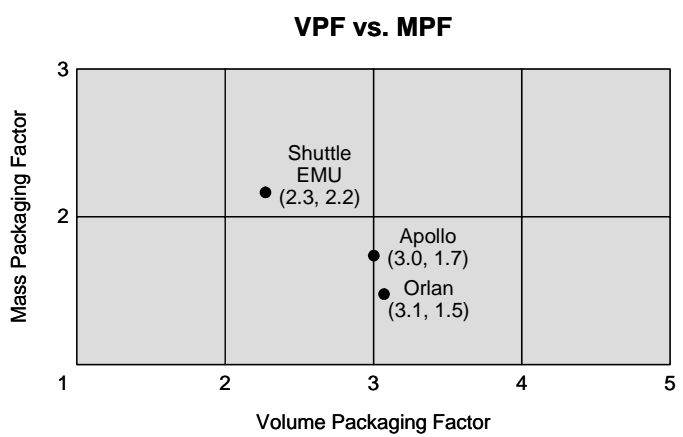

Figure 3 - $\mathrm{PLSS}_{\mathrm{PF}}$ vs. $\mathrm{PF}_{\mathrm{v}}$

\section{NASA In House Packaging Design Study}

An in-house design study $(3,4)$ was accomplished by NASA in the 1998 time frame to seek ways to reduce the weight of PLSS packaging, and at the same time, develop a packaging scheme that would make PLSS technology changes less costly than the current packaging methods. Experience over time shows that the exact technology change needed cannot easily be predicted. Consequently, there is a need to avoid packaging methods that embed a particular schematic; such as the Apollo and Shuttle embed the water based thermal control technology by making the water tank the structural backbone. If light weight is a consideration though, it is difficult not to use the structure of one or more of the components as the package structure. For this reason a major effort in the in-house design study was to be creative in how packaging for a PLSS is accomplished.

As part of the study, a functional decomposition was accomplished to define just what a PLSS packaging design is required to do. Overall PLSS packaging must:

\author{
Protect, Connect and Hold the PLSS \\ and its components together \\ internally and externally while \\ providing access to PLSS \\ components internally for \\ maintenance and for technology \\ change without extensive redesign \\ impact.
}

This is both a concise statement of PLSS packaging functionality and a statement of a key requirement to allow low cost schematic flexibility. During the study, 15 concepts were generated to satisfy the above functional statement, and using two rounds of the Decision Matrix method, the most promising concepts were reduced to three. No further progress could be made using the Decision Matrix method to discriminate between the remaining three concepts, so it was decided to build mock-ups of each concept. This was done and followed by another round of the Decision Matrix method based on the experience gained from building the mockups. From this effort, the final concept was decided to be a combination of two of the three concepts. This concept is called the Flex PLSS and was the starting point for this study.

\section{DISCUSSION}

\section{WORK PLAN AND ACCOMPLISHMENTS}

The work plan was divided into two major tasks. The first task is to bring the advanced space suit integrated Flex PLSS concept from the current state of development to a preliminary design level and build a proof of concept mockup of the resulting design. The second task is to "Design the Design Process" by documenting the methods and tools that will speed up and reduce the cost of the initial design and any subsequent redesigns of the PLSS packaging resulting from technology change.

\section{Task One - Flex PLSS to PDR Verified Via Mockup.}

The work plan for the first task was laid out to incorporate best design processes with an emphasis on fostering creativity. The challenge to remove $2 / 3$ of the weight of a system would require the team to be very creative. This expectation was fulfilled in the progress of this study. Knowing that "creativity favors the prepared mind" required that we understand the problem well; we planned and put considerable effort into defining the problem. Planned for that effort were subtasks to review the prior work, describe the PLSS interfaces, update the functional decomposition from the prior work, generate mini specs for each of the functions, develop evaluation criteria and, when all of that work was done, hold a formal problem definition review to assure all of the team had a good definition of the problem to base their creativity upon. This work was accomplished as planned. 
With the defined problem in mind, the plan for task one next focused on the creative process of generating ideas. First ideas were generated for satisfying the functions identified in the functional decomposition. Then assembling these function satisfying ideas into complete packaging solutions was planned and accomplished. As the concepts were generated the plan included documenting how each concept satisfied the functions including sketches of physical arrangement and failure mode and effects analysis. The plan laid out a process to define each concept to sufficient detail to be properly evaluated and each concept to the same level of definition so that the evaluation process would not have to deal with concepts at different levels of definition. This work was accomplished with the difficulty being faced of working hard to get all of the concepts to the same level of definition. The plan assumed three concepts would be generated by this process. In fact ten good concepts were generated.

The importance of generating good concepts can be seen from the following considerations. To meet the mass reductions stated, the goal is to reduce the $P F_{m}$ from the current 2.2 for the Shuttle EMU down to 1.24, an $80 \%$ reduction in PLSS packaging mass. This goal was chosen to bring the PLSS carry weight down to 65 pounds to be as close as possible to the $30 \%$ of crewperson mass mentioned above. This is a critical target because the mass packaging factor, along with the total mass of the PLSS determine the allowable mass of the life support. Since the total mass of the PLSS is targeted to be 65 pounds, this leaves only 52 pounds for components if the mass packaging factor target can be met. As the packaging factor increases, precious pounds of components are eliminated to maintain the overall mass (see Figure 4). Hence, the program risk increases significantly as packaging factors increase because it becomes much more difficult to meet the basic life support functional requirements.

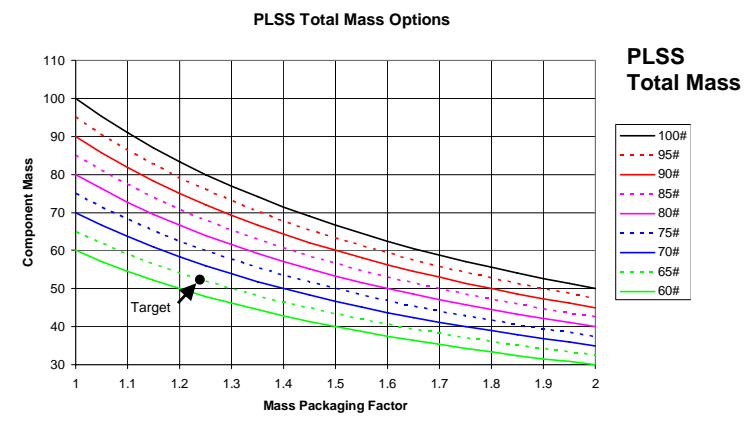

Figure 4 - PLSS Total Mass Options

Based on the documentation of the concepts generated, the plan proceeded to evaluating and down selecting. The planned process for the evaluation was the Decision Matrix method which evaluates the concepts against the criteria generated earlier in a manner suited for the stage of concept definition that can reasonably be expected at this early stage of concept development. This process was used and the evaluation brought the number of concepts under consideration from ten to three.

After completion of the first evaluation effort it was apparent that a second concept generation evaluation and down select effort would be required since none of the concepts generated during the first round met the weight goals. For the second round of concept generation, three PLSS packaging teams were assigned to each develop a unique concept. The teams were comprised of Hamilton Sundstrand Windsor Locks (HSWL), Hamilton Sundstrand Houston (HSMS), and NASA.

The HSWL concept, lacking only final thermal and stress analyses, had a calculated $\mathrm{PF}_{\mathrm{m}}$ that was $1.64,167 \%$ higher in packaging mass than the 1.24 target would allow. This was an unacceptably high factor. Worse, it was realized that both the HSMS and NASA concepts, though at very early design phases, would be around the same 1.64 value.

Since having all three teams miss the target by such a gross margin would amount to failure, a recovery plan was quickly developed. HSWL would maintain their concept since it was nearly complete, although they did have time to make minor adjustments to their protection mechanism. HSMS would alter their concept as needed to try to meet the target as much as possible. NASA meanwhile was tasked with the most radical work-plan, scrapping their initial concept and starting from scratch with the overarching objective of meeting the weight target.

An effective way to tackle the weight packaging factor problem was to divide it into its three primary constituents: protection, hold, and connect (see Figure $5 A, B, C)$. This simplifies the analysis by allowing the team members to concentrate on one of the areas at a time, thus making it much easier to understand and develop solutions.

Based on the HSWL design, protection accounted for $58 \%$ of the packaging mass. The NASA team therefore decided to concentrate much of its effort in this area. A gasbag protection system was developed which reduced the mass by $68 \%$ compared with the HSWL concept. 

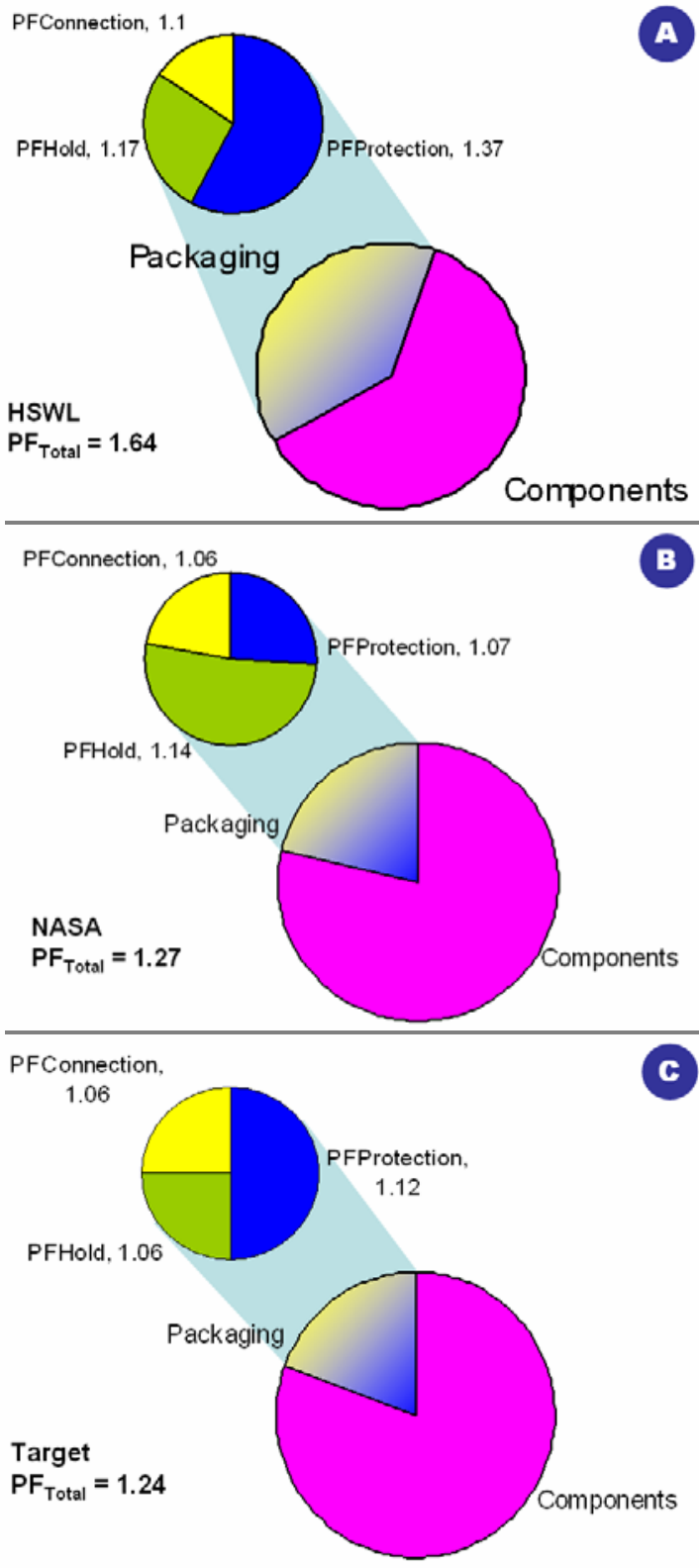

Figures 5: A, B, C -Packaging Factor Constituents and Progression

In the end, the NASA team was the closest to meeting the $1.24 \mathrm{PF}_{\mathrm{m}}$ target, coming within $13 \%$ of the packaging mass target (see Figure 6). The HSWL team was able to make a significant $\mathrm{PF}_{\mathrm{m}}$ improvement by making minor improvements to the protection mechanism.

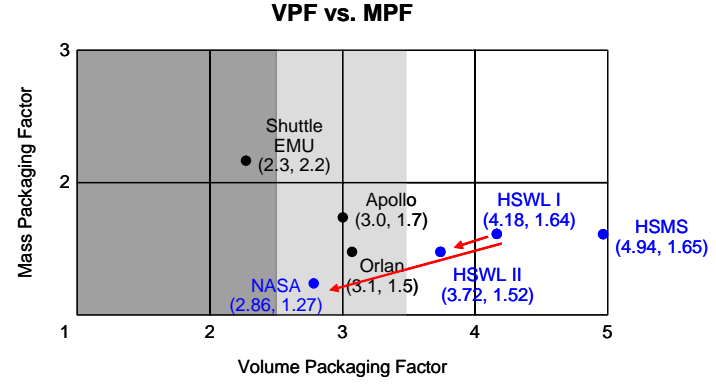

Figure 6 - PLSS $\mathrm{PF}_{\mathrm{m}}$ vS. $\mathrm{PF}_{\mathrm{v}}$

A significant difference between the NASA concept and the other two concepts were readily evident by simply looking at the exteriors (see Figure 7). The NASA concept was considerably more compact.

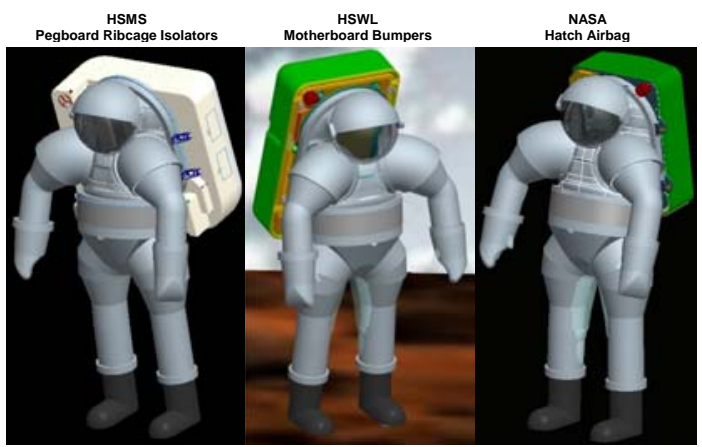

Figure 7 - Final Three PLSS Concepts

Although it was recognized ahead of time that all of the concepts did not meet the weight target, a second round of evaluation was accomplished using the Decision Matrix method. This evaluation was held to drive out the weak and strong points of each concept to guide future work since none of the concepts met all of the goals set out at the start of the effort. Two concepts did not approach the weight target but the concept that did approach the weight target sacrificed flexibility for technology change and in use maintenance to get as close as it did to the weight target. The lightest weight concept was selected for further work. Subsequent to that selection, the HSWL team worked to improve the maintainability of the concept.

A mock up of the selected concept was included in the plan to validate the concept and to assure the concept was indeed realizable. The mockup will be used to evaluate how far from the flexibility for technology change target and the in use maintenance target the final concept actually is. These issues are very difficult to asses without a physical realization with which to work. 


\section{TASK ONE LESSONS LEARNED}

\section{Monuments}

System monuments (hardware that prevents the rapid and easy redesign of a system to accept new components) are the greatest roadblock to technology and mission adaptability. For example, on the EMU, the water tank and valve modules perform multiple functions (Protect, hold, connect) and would have to go through extensive redesigns if moderate changes were made to that PLSS (Figure 8).

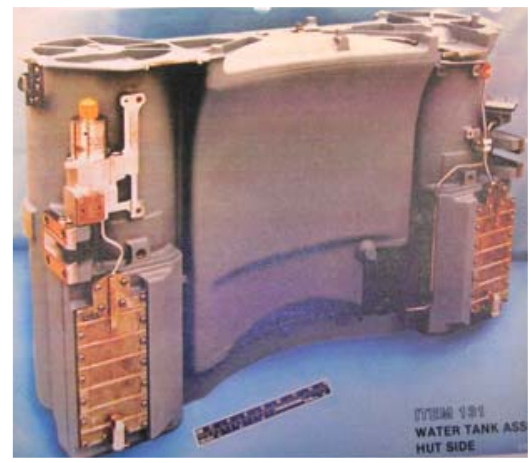

Figure 8 - EMU Monuments

The conceptual relationship between technology adaptability, mission adaptability (both of which can be termed as flexibility), ease of maintenance and the reduction in the number of monuments used can be mapped (Figure 9).

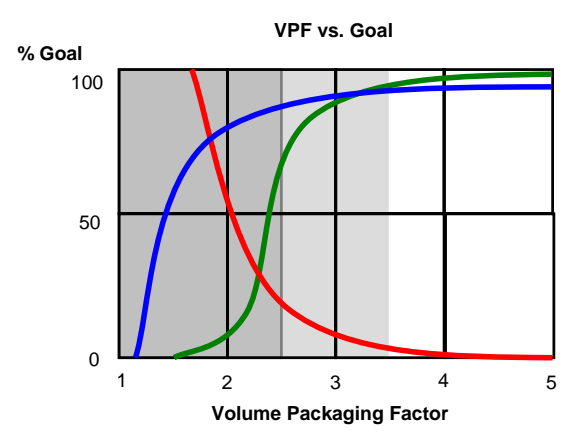

Figure 9 - Flexibility, Maintainability and Number of Monuments vs. Volume Packaging
If the number of components in monuments in the packaging concept is large, the mission and technology adaptability goal is low but the volume is very low. The low volume can make the maintainability goal hard to reach so that goal is possibly low as well when the number of monuments is high. In order to achieve the desired flexibility and maintainability, monuments must be avoided. Furthermore, it was evident that as the packaging size is reduced, monuments need to be introduced as they are the only design solutions that would allow for the small packaging size (the Shuttle EMU is an example). Thus, it was determined that from a flexibility standpoint, the volume was the critical metric, not the mass (see figure 10).

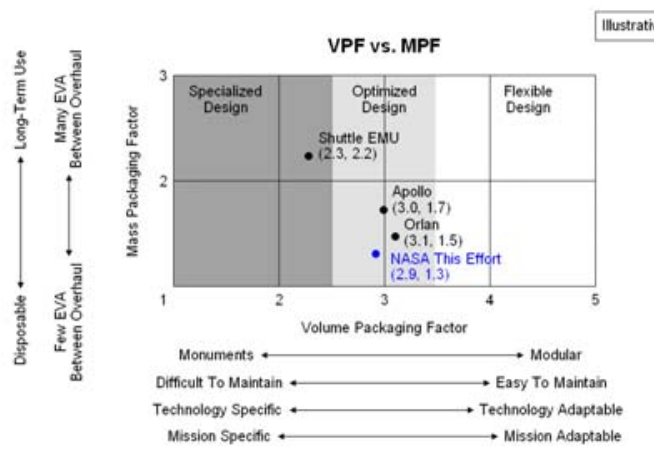

Figure 10 - PLSS Flexibility

\section{Selection Process}

How could we have had three designs that were all too heavy to accomplish the mission, and not recognize it until late in the process? That was a troubling question. We applied the typical filter process that is often used to narrow down the concepts from many potential candidates, to a few promising ones, to finally a winning concept. Each filter during the selection process becomes progressively more detailed.

For example, if the objective was to get a boat across the Atlantic, the selection process should use three filters to choose the best design to do the job (see Figure 11). In this case, the sail boat is the winning design because it can actually get across the Atlantic, and it is the lowest cost, simplest, lightest solution that meets all of the requirements. 


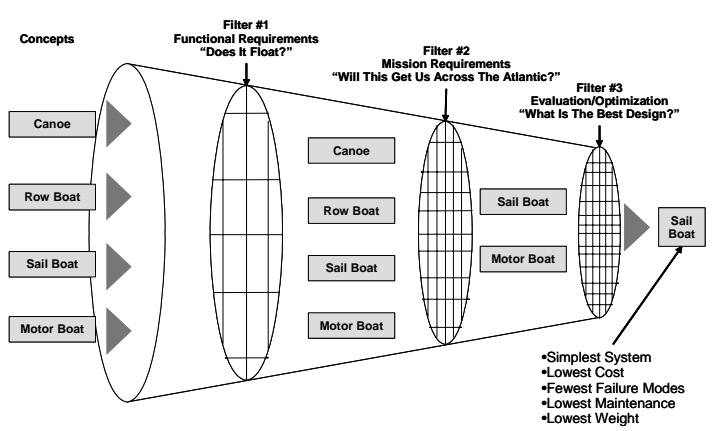

Figure 11 - Filter Selection Process Example 1

However, if filter \#2 is missing, then the winning design can be quite different (see Figure 12). In this case, the canoe is the winning design because it is the lowest cost, simplest lightest solution even though it cannot actually meet the requirement to cross the Atlantic. By skipping the middle filter and jumping directly into a deep-level analysis, the end goal can be lost in a search for optimization.

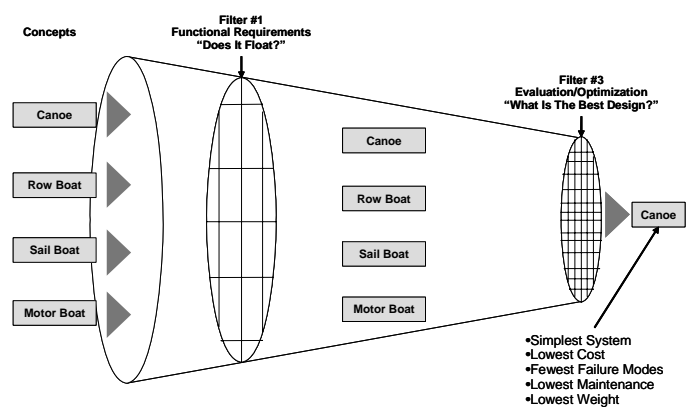

Figure 12 - Filter Selection Process Example 2

This is what was happening in the early stages of the second round process. There was a very detailed analysis being done on three concepts, which, at a high level, could not actually work because they are too heavy. In the final selection process, the team ensured the middle filter was not ignored.

The team did not have a sense of the true importance of requirement weighting factors for the different figures of merit; we did not go through the team consensus process. We needed to get started on developing concepts, so to save time the team individually ranked the criteria and the average ranking was used. Had we discussed the ranking to develop a team consensus it is very likely that a low mass packaging factor would have been identified as an absolute (must meet) requirement.

\section{Mass Reduction}

Mass reduction techniques can be broken into two categories. 1) Breakthrough design concepts (examples are: the gasbag outer cover, the combined base plate / hatch with through bolt mounted LRU's. 2) Detail part weight optimization. This technique involves reviewing and trading, material selection, minimizing factors of safety, changing requirements, reducing wall thickness, etc. This needs to be done for every detail part, starting with the parts that will give the greatest weight reductions. The impact of another of the detail techniques, minimizing volume and surface area became obvious in this effort. The volume and surface area of the PLSS contributes to the overall mass. Hence, it is important to manage volume and surface area in order to minimize the PLSS mass (see Figure 13).
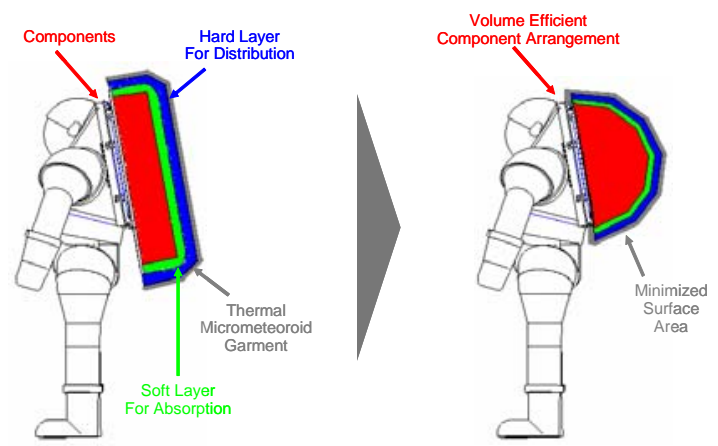

Figure 13 - Volume \& Surface Area Reduction

\section{Task Two - "Design" the Design Process}

The Constellation Program plans an evolutionary process to use the lunar missions to learn the best way to go to Mars. This planned evolving of systems and processes makes it key to plan for change. The goals of the flex PLSS design process task are:

- to reduce the design schedule,

- reduce the redesign schedule

- optimize the design tools by making effective use of readily available design tools or develop design tools if they are not available

- document lessons learned and design methodologies.

As task one unfolded, the team responsible for task two remained in a support and observing role so as to have the freedom to capture and optimize the design process.

DESIGN PROCESS The major steps of the process that emerged are:

1. Get stake holds to agree on a common set of requirements and schematic. They need to be as complete as possible and written down. Change control is needed.

2. Develop concept layout - This can make or break the process. The designers need the most insight during this phase of the job; going down the wrong path (concept doesn't work) at this stage will cost you dearly in the detail design or production 
stage. This is where more system level oriented designers can really shine. The team needs to develop the right concept by performing quick/rough analysis that shows that the concept is acceptable in the following areas: placement of components (cg, maintenance, line runs, interfaces, etc), thermal, structural, envelope and interfaces, weight, failure modes. The concept needs to be reviewed and approved by the functional groups and customer before detail design starts if the program is committed to containing cost growth. An early mock up is important at this point where the human factors of maintenance can be assured by key stake holders such as flight crew. This can run in parallel with early detail design but careful management is required to assure detail design does not progress very far without the knowledge captured from the mock up effort.

3. Detail design and layout- once the concept is approved detail design begins. Instead of rules -of thumb and quick analysis that were used in the concepting phase, each component and the assembly is completely designed with full analysis as required. This is where more detail oriented designers can really shine. Analysis tools replace rules-of- thumb and technical experience. Tolerance studies replace estimates of how much room is needed get the parts to fit together. A significant part of the packaging task during this phase is the interface control between the customer and/or suit and the package and the internal components. These interfaces not only include geometric interfaces but also functional interfaces such as electrical, thermal, deflections, pressure drop/fittings, load transfer, and EMI. This is the point in the program where the definitions information is captured and documented which will allow the technology driven modifications to be done with a minimum of effort. 4. Final design review and detail drawing approval - The key stake holders (customer, users, operators, maintainers, etc.) and the in house functional groups (Systems, Materials, Mechanical design, Electrical Design, Drafting, Safety and Reliability, and Operations) review the design (layout and detail drawings) from the perspective of their expertise. Each group needs to understand the design and use their experience to find potential problems early enough to allow changes within the allotted schedule. This early understanding also assures the optimum functional input to come early in the schedule. Formalizing this process is a must.

5. Technology change driven redesign - New mission requirements will drive technology change. To minimize the design effort required to incorporate new technologies these recommendations should be followed. A) The initial design requirements and therefore the final design configuration must incorporate features allowing for technology change. B) The design must be documented such that any redo's have available to them the analysis, test results and all geometric definition of the original design. This should cumulate in a summary document which contains an index which defines the location of all back up data, a summary of top level performance margins, a list of key personnel to see for historical back-up, and a section for changes that will occur due to testing, failures, engineering changes, and reworks. C) A total unit with exact representation (a full up development PLSS of known fidelity) available to the detail designers. In addition to the above a highly desirable feature would be testing to the limit during initial development so that any requirement changes due to the new technology could be evaluated real time. It is important that the design, analysis, testing be well documented so that design can be redesigned with the lowest cost and schedule.

TOOLS SECTION DURING the concept phase Pro-e $\odot$ and Mechanica $\odot$ will be used to rough out the concept. It is important to keep the modeling simple, for example: don't put in fillet radii unless needed to show an important concept idea. Model the major structural members with limited detail. When doing Mechanica॰ stress analysis in this time frame, look for stress in major structural members, don't worry about radii and local stress concentrations, they will get worked out during the detail design phase. Mathcad $\odot$ and MS EXCEL $\odot$ spreadsheets are also used in the concept and detail design stage for such varied tasks as weight evaluations and bookkeeping and automation of trade study iterations.

Dytran॰ is used for the non-linear analysis of the gasbag. The geometry changes very drastically during an impact event, so Nastran॰ FEA will not work. Prototype gasbags need to be made and tested to validate the Dytran॰ model, once validated the model can be used to run many" what if" cases to assure that the concept is feasible.

During the detail design phase the same tools are used in more depth. Advanced designers may use NASTRAN instead of Mechanica๑ for analysis. Nastran๑ is more versatile but it requires more training to use

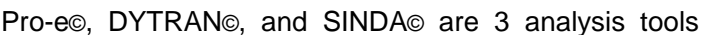
that should be used. We still need to find ways of integrating tools so that the results can be fed back to the designer quicker.

LESSONS LEARNED: By watching the group brainstorm concepts, a list of lessons learned were captured when errors and redo's were encountered. By following lessons learned, mistakes will not be repeated. Over time, the lessons learned section of the Flex PLSS design process guidelines will continue to grow.

Spend the time to get the concept right up front, before detail design is started. The more detailed the concept or design gets before it is found to be unacceptable, the more costly will be the recovery effort. To prove out a new idea/concept, either a first cut analysis or a feasibility test is needed. If the basic concept depends on a unique idea a test must be run before concept approval. 
In the conception phase the program needs experienced, inventive, engineers that won't get bogged down in detail that is not needed until later in the program. In the detail design phase the program needs detail orientated designers.

The weight target is very difficult to meet. So develop a weight control plan at the beginning of the program with estimated or calculated weights so that corrective actions can be taken as soon as possible.

The concepts generated resulted in unacceptably high component operating temperatures. This is the result of not having thermal analysis personnel available early in the concept phase. The complexity of the heat transfer within the PLSS prevented the designer from doing his own analysis. The lesson learned is to plan ahead.

Document the importance of the key requirements and re-evaluate periodically to assure program success, or direct design changes as early as possible.

No silver bullet was found that drastically reduced the present time it takes to redesign the PLSS to incorporate new technology. The tools and documentation created during the initial design will help reduce redesign time as will the knowledge gained from designing it the first time. Well-validated analysis models will reduce redesign time and reduce the development and certification testing required on the redesigned PLSS. Planning ahead of time for technology changes that are likely to be incorporated will also help. This planning includes grouping functional components together that may be replaced and trying to reserve envelope and interfaces for the new technology.

Task Two Results

The design guideline document that will result from Task Two will reduce the overall cost and schedule of designing PLSS packaging. The design guidelines contain good procedures, and the latest proven design and analysis tools. Most findings have been well known on past jobs but are difficult to implement in the "heat of battle" of "today's problem". Good planning, follow through, and value added oversight continue to be important to maintaining design excellence, cost, and schedule. Well-defined design and system requirements are also necessary in order to reduce redo's that increase cost and schedule.

\section{CONCLUSION}

Taking two thirds of the mass out of a space suit system is no easy task. Much creativity is needed and a difficult balance must be struck with competing requirements such as maintainability or ease of incorporating new technology. We have made progress in understanding the packaging weight problems that need to be solved and have developed a new packaging concept that requires further development. Proper use of the latest proven design tools and following proven design and development procedures can significantly reduce cost and schedule. The challenge to reduce on back mass still exists and creativity of the team will be required to prevent the reduction of the EVA operating time for the final design.

\section{References}

1. Packaging Factors for Portable Life Support Subsystems Based on Apollo and Shuttle Systems, Gretchen A. Thomas, $23^{\text {rd }}$ ICES, Colorado Springs, Colorado, July, 1993, 932182.

2. Space Suit Life Support System Packaging Factors, I.P. Abramov, R. K. Sharipov, A.I. Skoog, and Nikolaus Herber, 24 ${ }^{\text {th }}$ ICES, Friedrichshafen, Germany, June, 1994, 941380

3. PLSS Packaging Design, Michael Rouen, $29^{\text {th }}$ ICES, 1999-01-1994.

4. Advanced Spacesuit Portable Life Support System Packaging Concept Mock-Up Design \& Development, Mary K. O'Connell, Howard G. Slade, Richard G. Stinson, $29^{\text {th }}$ ICES, 1999-01-1995.

\section{CONTACT}

Michael Rouen is a 41-year veteran mechanical engineer at the Johnson Space Center. His background is in Portable Life Support System and EVA System design. $\mathrm{He}$ may be contacted at michael.n.rouen@jnasa.gov

\section{DEFINITIONS, ACRONYMS, ABBREVIATIONS}

EMU: Extravehicular Mobility Unit (the current shuttle space suit).

FEA: Finite Element Analysis

HSWL: Hamilton Sundstrand Windsor Locks

HSMS: Hamilton Sundstrand Houston

ISS: International Space Station.

LiOH: Lithium Hydroxide, a non-regenerable Carbon Dioxide absorbent chemical.

NASA: National Aeronautics and Space Administration

METOX: The name given to a metallic oxide regenerable Carbon Dioxide absorbent system used to replace $\mathrm{LiOH}$ for ISS use.

Packaging: PLSS packaging is defined by the following ground rules.

- Any item performing a major, useful life support function is a component to be packaged and is not packaging. 
- Harnesses, connectors, switches, brackets, wiring, and plumbing are packaging.

- Structure is packaging, even in such special cases as the Shuttle valve module housing.

PDR: Preliminary Design Review

PLSS: Portable Life Support System
SOP: Secondary Oxygen Pack

LRU: Line Replaceable Unit

HSWL: Hamilton Sunstrand, Windsor Locks

CEV: Crew Exploration Vehicle

CG: Center of Gravity 